\title{
Evaluation of two coproscopic techniques for the diagnosis of schistosomiasis in a low-transmission area in the state of Minas Gerais, Brazil
}

\author{
Liliane Maria Vidal Siqueira', Paulo Marcos Zech Coelho', Áureo Almeida de Oliveira', \\ Cristiano Lara Massara², Nídia Francisca de Figueiredo Carneiro ${ }^{3}$, \\ Anna Carolina Lustosa Lima', Martin Johannes Enk1,4/+
}

\footnotetext{
'Laboratório de Esquistossomose 2Laboratório de Helmintologia e Malacologia Médica, Instituto de Pesquisas René Rachou-Fiocruz, Av. Augusto de Lima 1715, 30190-002 Belo Horizonte, MG, Brasil ${ }^{3}$ Centro de Controle de Zoonoses, Montes Claros, MG, Brasil ${ }^{4}$ Laboratório de Parasitologia, Universidade Vale do Rio Doce, Governador Valadares, MG, Brasil
}

This population study, which evaluated two parasitological methods for the diagnosis of schistosomiasis mansoni, was performed in a low-transmission area in Pedra Preta, Montes Claros, Minas Gerais, Brazil. A total of 201 inhabitants of the rural area participated in this research. Four stool samples were obtained from all participants and analysed using the Kato-Katz method (18 slides) and a commercial test, the TF-Test ${ }^{\mathbb{R}}$, which was performed quantitatively. The data were analysed to determine prevalence, the sensitivity of the diagnostic methods, the worm burden and the definition of the "gold standard", which was obtained by totalling the results of all samples examined using the Kato-Katz technique and the TF-Test ${ }^{\circledR}$. The results showed that the prevalence obtained from the examination of one Kato-Katz slide (the methodology adopted by the Brazilian control programme) was 8\% compared to $35.8 \%$ from the "gold standard", which was a 4.5-fold difference. This result indicates that the prevalence of schistosomiasis in so-called low-transmission areas is significantly underestimated.

Key words: Schistosoma mansoni - schistosomiasis - diagnosis - control - low-transmission area - Brazil

An accurate diagnosis of Schistosoma mansoni infection is the basis for defining the prevalence and understanding the epidemiology and morbidity of the disease. When the focus is on transmission control, the assessment of cure after therapeutic interventions and the selection of adequate control measures for the reliable detection of schistosomiasis infections gain even more importance (Berhe et al. 2004). In general, the disease is diagnosed using stool examinations; however, due to the relatively low sensitivity of these methods, the true prevalence of the disease has been underestimated, with important consequences for disease control in low-transmission settings (De Vlas \& Gryseels 1992). Regarding intestinal schistosomiasis, the Kato-Katz method (Katz et al. 1972) is the diagnostic technique of choice (WHO 1993). The Kato-Katz method has been extensively used under conditions of precarious laboratorial infra-structure, mainly because of its low operational costs, practicality and ability to be quantitative. Nevertheless, after specific treatment and in individuals with a low worm burden and those in low-prevalence areas, this technique has been found to be less sensitive (Kongs et al. 2001, Ferrari et al. 2003, Lin et al. 2008, Zhang et al. 2009). Studies con-

Financial support: FAPEMIG, CNPq, FIOCRUZ

+Corresponding author: marenk@cpqrr.fiocruz.br

Received 20 April 2011

Accepted 20 September 2011 ducted in areas with those characteristics have demonstrated a direct relationship between the number of slides analysed and the infection rate of the disease (Enk et al. 2008). However, the preparation of multiple slides and samples for each individual results in logistical difficulties, thus neutralising the operational advantages of the technique and stands in contrast to the examination of a single slide per individual using the Kato-Katz method for control campaigns (Teles et al. 2003).

Therefore, the individual's low worm burden plays an important role in the choice of diagnostic methods, which tend to become more complex as endemicity levels decline (Bergquist et al. 2009). Another consequence of this is an increased disagreement between the results obtained using different diagnostic techniques.

In the present study, the TF-Test ${ }^{\mathbb{}}$, an innovative method based on formalin-ethyl acetate centrifugation of three faecal samples collected on different days and the Kato-Katz technique were compared and the diagnostic accuracy of both methods was evaluated for schistosomiasis diagnosis in a low-transmission area.

\section{SUBJECTS, MATERIALS AND METHODS}

Study area and population - This study was performed in the communities of Buriti Seco and Morro Grande in Pedra Preta, a little village in a schistosomiasis-endemic area in the rural region of Montes Claros, state of Minas Gerais (MG), Brazil. This area was chosen based on the population not being treated for schistosomiasis and because it shows a low migration index (fixed resident population). In addition, according to data provided by the Montes Claros Control Centre of 
Zoonosis, an infection rate of $12 \%$ was found in 2005 . The populations of these communities were included in this study. A total of 201 individuals (93 women and 108 men) between one-96 years old were invited to provide stool samples for examination.

Diagnostic methods - Four stool samples per individual were collected on four consecutive days using $100-\mathrm{mL}$ plastic pots and identified using the name and number of the participant and the identification of the residence.

Kato-Katz method - The Kato-Katz method is a quantitative technique that allows for a good visualisation of $S$. mansoni eggs in the faeces. This method was performed using 18 slides, which were prepared as follows for each participant: 12 slides of the first sample and two slides each of the second, third and fourth sample in a total of $750 \mathrm{mg}$ of faeces $(18 \times 41.7 \mathrm{mg})$ (Fig. 1).

Quantitative TF-Test ${ }^{\circledR}$ - The TF-Test ${ }^{\circledR}$ is primarily a qualitative method, according to the manufacturer's protocol (Immunoassay Ind \& Com SA, São Paulo, Brazil). Each of the first three samples was passed through a nylon mesh and $500 \mathrm{mg}$ was quantified in metal plates. Each $500 \mathrm{mg}$ portion was transferred to a tube of the kit containing preservative solution $(10 \%$ formalin) and processed using $3 \mathrm{~mL}$ ethyl acetate and a drop of neutral detergent. In the first experiment, the three tubes containing the samples collected from consecutive days were connected to the centrifuge tube and centrifuged at $500 \mathrm{~g}$ for $2 \mathrm{~min}$. The supernatant was discarded and the total sediment was re-suspended in $0.85 \%$ saline solution and analysed using optical microscopy (Gomes et al. 2004) (Fig. 2). In the second approach focusing on the direct comparison of the same amount of stool for each method, the results of 12 slides of the Kato-Katz method $(12 \times 41.7 \mathrm{mg}=500 \mathrm{mg})$ were compared to those of the TF-Test ${ }^{\circledR}$, which was modified using $500 \mathrm{mg}$ of the faecal material from the first sample of 54 participants (Fig. 1).

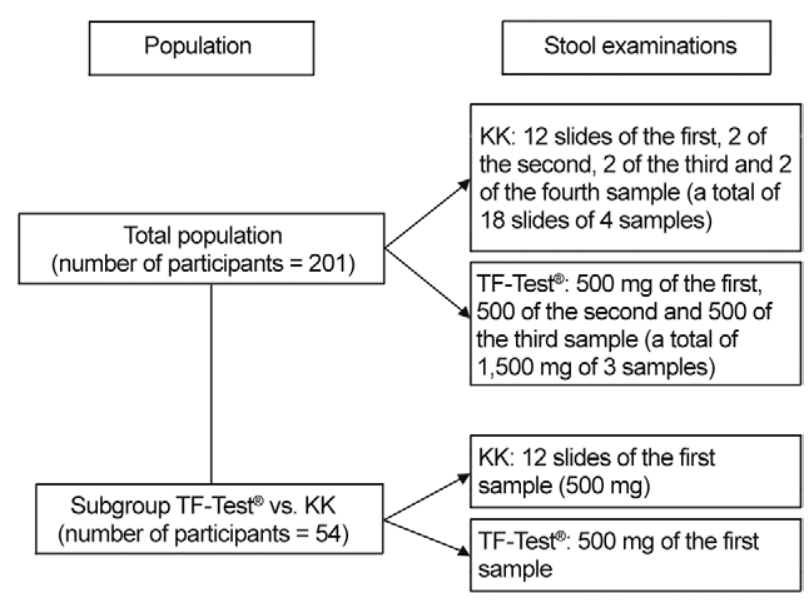

Fig. 1: flowchart of stool examinations carried out among the study population of Pedra Preta, Montes Claros, Minas Gerais, Brazil. KK: Kato-Katz method.
Intensity of infection - The worm burden was determined as eggs per gram of faeces (epg). The value for the individual intensity of infection was obtained according to the Kato-Katz method using the arithmetic mean of eggs found on each slide multiplied by 24 because each Kato-Katz slide has a capacity for $41.7 \mathrm{mg}(41.7 \times 24=$ $1,000 \mathrm{mg}$ or $1 \mathrm{~g}$ ). The number of epg was calculated according to the TF-Test ${ }^{\mathbb{B}}$ by multiplying the number of eggs detected in the total sediment by $2 / 3$ because this technique utilises $1.5 \mathrm{~g}$ of faeces $(1.5 \times 2 / 3=1 \mathrm{~g}$ of faecal material). The infection intensity at the population level was obtained by calculating the geometric mean of egg counts of positive participants.

Statistical analysis - SPSS version 15 (IBM, Chicago, USA), version 15 , was used for statistical analysis. The chi-square test was applied for comparisons among proportions, with a significance limit established at $\mathrm{p}<0.05$.

The "gold standard" was determined from the sum of all positive results obtained using both methods. This value was also used as a reference for the determination of the diagnostic accuracy of the techniques.

The prevalence of schistosomiasis infection was obtained from the number of positives determined using the "gold standard" and divided by the number of all participants.

The positivity rates of the different approaches using the Kato-Katz technique and the other parasitological method were calculated by dividing the number of positives detected in the different combinations of the number of slides or samples by the number of all participants.

The sensitivity was calculated with $95 \%$ confidence intervals $(95 \% \mathrm{CI})$.

The level of diagnostic agreement between different diagnostic approaches and the "gold standard" was determined using the kappa coefficient. According to Landis and Koch (1977), the concordance is bad when the kappa coefficient is lower than 0.20 , weak between 0.21-0.40, moderate between 0.41-0.60, good between 0.61-0.80 and excellent when higher than 0.81 .

Treatment of the positives - All participants who were positive for schistosomiasis were treated with praziquantel in a single dose of $60 \mathrm{mg} / \mathrm{kg}$ for children and

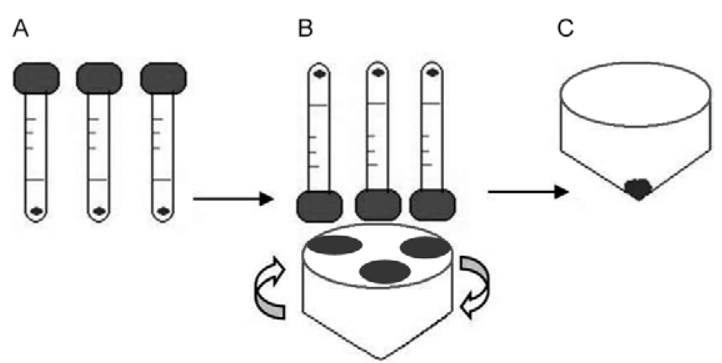

Fig. 2: illustrative scheme of the TF-Test ${ }^{\circledR}$ kit. A: three collecting tubes containing $500 \mathrm{mg}$ of faecal material of three different samples conserved in $10 \%$ formol solution; B: adaptor for the centrifuge to attach the collecting tubes; $\mathrm{C}$ : centrifugation tube with the sediment ready for transfer to slides and examination. 
$50 \mathrm{mg} / \mathrm{kg}$ for adults. Infections with other helminths were treated with a single dose of $400 \mathrm{mg}$ albendazole, as recommended by the Brazilian Ministry of Health.

Ethics - This project was approved by the Ethical Research Committee of the René Rachou Research Institute-Fiocruz (CEPSH/CPqRR 03/2008) and the National Brazilian Ethical Board (784/2008, CONEP 14886). The study objectives were presented and explained to all participants and written consent was obtained through signing a form before admission to this study.

\section{RESULTS}

From the results obtained using the two techniques, $72(35.8 \%)$ out of 201 participants were positive for $S$. mansoni, representing the "gold standard" value.

The positivity rate obtained by examining one KatoKatz slide was $8 \%$ (16 positives out of 201 participants). This rate demonstrates a significant underestimation of the disease prevalence by 4.5 -fold when compared with the prevalence of $35.8 \%$ obtained using the "gold standard".

The results of an increasing number of slides using the same stool sample revealed $16(8 \%)$ positives for one slide, 19 (9.5\%) for three, 25 (12.4\%) for six and 29 (14.4\%) for 12 slides. The sensitivity for each of these combinations of slides in relation to the "gold standard" is shown in Table I.

The well-accepted international standard of six Kato-Katz slides from three stool samples detected 43 positives $(21.4 \%)$. Reducing the number of slides per sample to one, i.e., one slide of each of the three samples, revealed 37 positives (18.4\%). The sensitivities for each approach are shown in Table II.

The combination of the first and second stool samples for a total of 14 slides detected 31 cases $(15.4 \%)$, indicating a sensitivity of $43.1 \%$ (95\% CI: 31.6-54.5). The combination of three stool samples for a total of 16 slides detected 53 positives (26.4\%), with a sensitivity of $73.6 \%$ (95\% CI: $63.4-83.8)$. The examination of 18 slides of all four samples revealed 58 positives (28.9\%), with a sensitivity of $80.6 \%$ (95\% CI: 71.4-89.7) in relation to the "gold standard".

The TF-Test ${ }^{\circledR}$ performed with three samples detected 32 (15.9\%) positive individuals for S. mansoni; 14 of these individuals were not identified using the KatoKatz method examining 18 slides.

Comparing the results obtained from the TF-Test ${ }^{\circledR}$ using 1,500 $\mathrm{mg}$ faecal material and six Kato-Katz slides of three different samples (2 slides of each) with the "gold standard" revealed a lower sensitivity of the TF-Test ${ }^{\circledR}$ $(44.4 \%)$. The sensitivity of the Kato-Katz method was higher at $59.7 \%$ (43/72), even using only $250 \mathrm{mg}$ of faeces (41.7 mg x $6=250 \mathrm{mg}$ ), i.e., six times less faecal material than that used in the TF-Test ${ }^{\mathbb{}}$. However, this difference was not statistically significant $(\mathrm{p}=0.067)$ (Table III).

Comparing the results obtained from three KatoKatz slides ( 1 of each sample) and the TF-Test ${ }^{\circledR}$ with the those of the "gold standard", sensitivity rates of 51.4\% (37/72) and 44.4\% (32/72), respectively, were detected; however, this difference was not statistically significant $(\mathrm{p}=0.404)($ Table IV).
TABLE I

Effect of increasing the number of slides of the same stool sample analyzed by the Kato-Katz method on the rate of positive individuals for Schistosoma mansoni and on the sensitivity in relation to the "gold standard"

\begin{tabular}{lcccc}
\hline & \multicolumn{4}{c}{ Number of slides } \\
\cline { 2 - 5 } & One & Three & Six & Twelve \\
Positivity rate (\%) & 8 & 9.5 & 12.4 & 14.4 \\
Sensitivity (\%) & 22.2 & 26.4 & 34.7 & 40.3 \\
\hline
\end{tabular}

\section{TABLE II}

Sensitivity and rate of positive individuals according to the "gold standard", the TF-Test ${ }^{\circledR}$

and different approaches utilizing the Kato-Katz method for the detection of Schistosoma mansoni infection in the locality of Pedra Preta, Montes Claros, Minas Gerais, Brazil

\begin{tabular}{lcc}
\hline & $\begin{array}{c}\text { Sensitivity } \\
(\%)\end{array}$ & $\begin{array}{c}\text { Rate of } \\
\text { positive individuals } \\
(\%)\end{array}$ \\
Technique & $100(72 / 72)$ & $35.8(72 / 201)$ \\
"Gold standard"a & $80.6(58 / 72)$ & $28.9(58 / 201)$ \\
KK (18 slides $=750 \mathrm{mg})$ & $814.4(29 / 201)$ \\
KK (12 slides = 500 mg) & $40.3(29 / 72)$ & $14.3(43 / 201)$ \\
KK (2 slides $/ 3$ samples) & $59.7(43 / 72)$ & $21.4(37 / 201)$ \\
KK (1 slide/3 samples) & $51.4(37 / 72)$ & $18.4(16 / 201)$ \\
KK (1 slide $=41.7 \mathrm{mg})$ & $22.2(16 / 72)$ & $8(16 / 201)$ \\
TF-Test ${ }^{\circledR}(1,500 \mathrm{mg})$ & $44.4(32 / 72)$ & $15.9(32 / 201)$ \\
\hline
\end{tabular}

a: "gold standard", the parasitological methods in association [18 Kato-Katz (KK) slides $=750 \mathrm{mg}$ and TF-Test $\left.{ }^{\circledR} 1,500 \mathrm{mg}\right]$.

TABLE III

Comparison between results detected by the TF-Test ${ }^{\circledR}$ and Kato-Katz method using six slides, two per each of three samples

\begin{tabular}{lcccc}
\hline & & \multicolumn{3}{c}{ Kato-Katz slides } \\
& & \multicolumn{3}{c}{$(2 \times$ of 3 samples $=250 \mathrm{mg})$} \\
\cline { 3 - 5 } & Positive & Negative & Total \\
TF-Test $^{\circledR}$ & Positive & 13 & 19 & 32 \\
$(1,500 \mathrm{mg})$ & Negative & 31 & 138 & 169 \\
& Total & 30 & 139 & 201 \\
\hline
\end{tabular}

The results obtained from the direct comparison of 500 $\mathrm{mg}$ faecal material from the same sample of 54 individuals between the TF-Test ${ }^{\circledR}$ and 12 Kato-Katz slides showed four (7.4\%) and eight (14.8\%) positive individuals for S. mansoni, respectively. Both techniques identified 10 positive cases and two of them were in concordance (Table V). 
To verify the diagnostic concordance, we calculated the kappa index for both methods in relation to the "gold standard", varying the number of slides analysed using the Kato-Katz method. Thus, the concordance obtained between the "gold standard" and one Kato-Katz slide was weak, moderate with 12 Kato-Katz slides and excellent with 18 slides. The TF-Test ${ }^{\circledR}$ showed a moderate concordance (Table VI).

Regarding geohelminths, $53(26.3 \%)$ individuals were positive for hookworms, eight (4\%) for Enterobius vermicularis and three (1.5\%) for Trichuris trichiura, as determined through the examination of 18 slides using the Kato-Katz technique. The TF-Test ${ }^{\circledR}$ was less efficient, detecting only six $(3 \%)$ positive participants for hookworms and no other helminths (Table VII).

Using both methodologies, the individual worm burden results showed $70(97.2 \%)$ individuals with a low worm burden (between 1-100 epg), of whom 24 presented only $1 \mathrm{epg}$. One positive individual (1.3\%) showed a medium worm burden with $156 \mathrm{epg}$ and another individual (1.3\%) showed a high worm burden with $555 \mathrm{epg}$. The epg results of each diagnostic method were analysed. All 32 positive cases detected using the TF-Test ${ }^{\circledR}$ presented a low worm burden: 23 individuals had an epg of one-10 and nine had between 12-47 epg. Eighteen slides analysed using the Kato-Katz method showed 56 (96.5\%) positive cases with a low worm burden (1-100 epg), one with a medium worm burden and one with a high worm burden. Of the 16 positive individuals detected using a single Kato-Katz slide of the first sample, 15 showed a low worm burden and one showed a high worm burden. The infection intensity at the population level according to the "gold standard" was 5 epg, with 18 Kato-Katz slides showing 6 epg and one Kato-Katz slide resulting in 57 epg. The TF-Test ${ }^{\circledR}$ revealed a mean worm burden of 6 epg.

Fig. 3 shows the prevalence obtained using the analysis of a single Kato-Katz slide and the value acquired for the "gold standard" stratified by eight age groups. The results from a single slide showed higher positivity rates for teenagers and juveniles, followed by a continuous decrease in the proportion of infected individuals among adults. The results of the "gold standard" showed, as expected, a general increase in the prevalence values for each age group and remarkably, high prevalence levels were maintained among 10-39-year-old individuals.

\section{DISCUSSION}

The conventional diagnosis for $S$. mansoni infection is performed using the Kato-Katz technique, which is recommended because it is quantitative, cheap and easy to perform (WHO 1993). However, in individuals with a low worm burden, it is difficult to detect eggs in faecal samples due to the low number of eggs eliminated, the daily variation in egg excretion and the analysis of a low quantity of faecal material ( $41.7 \mathrm{mg}$ of faeces) used in a single sample (Kongs et al. 2001). Because of these factors, this procedure, adopted by schistosomiasis control programmes, presents a significant underestimation of the true prevalence, particularly among individuals with a low worm burden and in areas of low disease transmission (Enk et al. 2008). The presence of $S$. mansoni
TABLE IV

Comparison between results for infection with Schistosoma mansoni detected by the TF-Test ${ }^{\circledR}$ and Kato-Katz method using three slides, one per each of three samples

\begin{tabular}{|c|c|c|c|c|}
\hline \multirow{3}{*}{$\begin{array}{l}\text { TF-Test }^{\circledR} \\
(1,500 \mathrm{mg})\end{array}$} & \multirow[b]{3}{*}{ Positive } & \multicolumn{3}{|c|}{$\begin{array}{c}\text { Kato-Katz slides } \\
\text { (1 x } 3 \text { samples) }\end{array}$} \\
\hline & & Positive & Negative & Total \\
\hline & & 6 & 26 & 32 \\
\hline & Negative & 31 & 138 & 169 \\
\hline & Total & 37 & 164 & 201 \\
\hline
\end{tabular}

TABLE V

Results for sensitivity and rate of positive cases in the direct comparison between the TF-Test ${ }^{\circledR}$ and Kato-Katz method using the same amount $(500 \mathrm{mg})$ of faecal material for the detection of infection with Schistosoma mansoni

\begin{tabular}{lcc}
\hline Technique & $\begin{array}{c}\text { Sensitivity } \\
(\%)\end{array}$ & $\begin{array}{c}\text { Rate of } \\
\text { positive cases } \\
(\%)\end{array}$ \\
\hline Kato-Katz $(12 \text { slides }=500 \mathrm{mg})^{80(8 / 10)}$ & $14.8(8 / 54)$ \\
TF-Test $^{\circledR}(500 \mathrm{mg})$ & $40(4 / 10)$ & $7.4(4 / 54)$ \\
\hline
\end{tabular}

TABLE VI

Diagnostic concordance of the Kato-Katz method and TF-Test ${ }^{\circledR}$ for the detection of Schistosoma mansoni in relation to the "gold standard"

\begin{tabular}{lcc}
\hline Technique & Kappa index & Concordance \\
\hline TF-Test $^{\circledR}$ & 0.498 & Moderate \\
Kato-Katz (1 slide) & 0.268 & Weak \\
Kato-Katz (12 slides) & 0.464 & Moderate \\
Kato-Katz (18 slides) & 0.844 & Excellent \\
\hline
\end{tabular}

TABLE VII

The prevalence of intestinal helminths according to 18 Kato-Katz slides among the population of Pedra Preta, Montes Claros, Minas Gerais, Brazil

\begin{tabular}{lccc}
\hline Helminths & $\begin{array}{c}\text { Positive } \\
(\mathrm{n})\end{array}$ & $\begin{array}{c}\text { Negative } \\
(\mathrm{n})\end{array}$ & $\begin{array}{c}\text { Prevalence } \\
(\%)\end{array}$ \\
\hline Ascaris lumbricoides & 0 & 201 & 0 \\
Hookworm & 53 & 158 & 26.3 \\
Enterobius vermicularis & 8 & 193 & 4 \\
Trichuris trichiura & 3 & 198 & 1.5 \\
Taenia sp. & 0 & 201 & 0 \\
Hymenolepis nana & 0 & 201 & 0 \\
\hline
\end{tabular}


eggs in stool shows a greater variation among slides of different samples than among slides of the same sample, thus indicating that the parasitological examination of samples on different days would be more appropriate for the estimation of more accurate prevalence (Utzinger et al. 2001). Therefore, negative parasitological examinations using a few samples and slides do not definitively exclude the possibility of false-negative results.

In a study performed in Ethiopia, Berhe et al. (2004) compared the results obtained from one, three and five Kato-Katz slides, which were prepared from a single faecal sample. The prevalence of $S$. mansoni infection was $31.3 \%$ based on a single slide, $45.7 \%$ in three slides and $52.1 \%$ in five slides. In the present study, similar findings were demonstrated, corroborating the work of these authors by showing an increase in positives concomitant with an increase in slides, as shown in Table I.

In our study, 12 slides of the first stool sample presented a $14.4 \%$ positivity rate. When two slides each of the second, third and fourth sample were examined, this rate increased from $15.4-26.4 \%$ and $28.9 \%$, respectively. Considering the results obtained, the analysis of three faecal samples seems to be the best choice because the variation in the number of $S$. mansoni eggs discharged in the faeces on different days is accounted for using this approach (Barreto et al. 1990, Engels et al. 1996, Kongs et al. 2001, Enk et al. 2008). However, this approach will certainly present important logistical problems for surveys performed on a large scale, neutralising all of the operational advantages of this method.

In this context, it is worth noting that in our study, the number of positive participants identified using two slides of the first three samples was 43 (21.3\%), indicating a 1.5-fold higher detection rate than 12 slides obtained from a single sample, which showed 29 (14.4\%) infected. This result indicates that the diagnostic sensitivity increases more with an increase in the number of different faecal specimens than with an increase in the number of slides from the same sample.

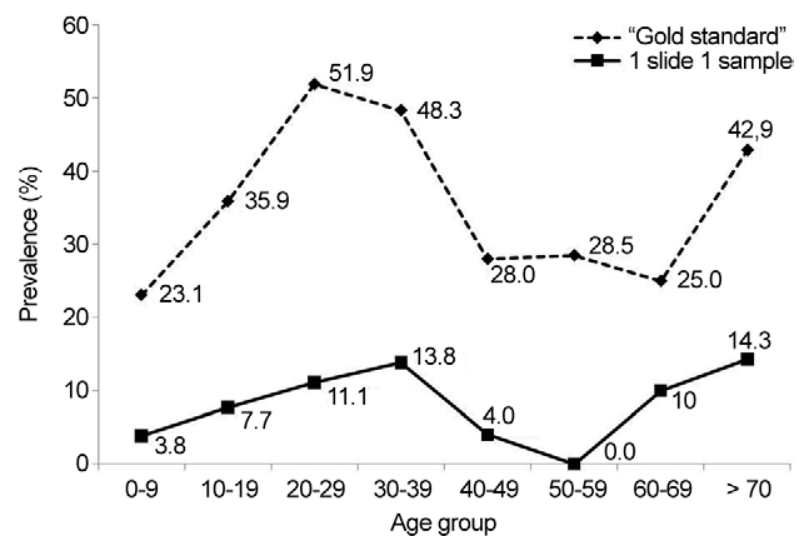

Fig. 3: prevalence of infection with Schistosoma mansoni according to age groups detected by one Kato-Katz slide of a single stool sample and by the "gold standard" among the participants of the locality of Pedra Preta, Montes Claros, Minas Gerais, Brazil.
In a study performed by Enk et al. (2008) in the district of Chonim de Cima, Governador Valadares, MG, comparing the methods of Kato-Katz and Blagg (Blagg et al. 1955), an increase in the prevalence rate from 13.8$35.4 \%$ was observed. In the current work, comparing the prevalence obtained using a single Kato-Katz slide (routine procedure of the control programmes in Brazil) with that of the "gold standard", an increase from $8-35.8 \%$ (4.5 times) was observed. Our results corroborate the data obtained by Enk et al. (2008), indicating that the prevalence of schistosomiasis (obtained using a single Kato-Katz slide) has been significantly underestimated in areas considered of low endemicity.

Moreover, as shown in Fig. 3, the distribution of prevalence stratified by age groups using a Kato-Katz slide did not follow the model of the standard prevalence curve, with a peak among children and juveniles followed by a continuous decrease. According to the results obtained using the "gold standard", our data showed high levels of prevalence continuing throughout adult life. A plausible explanation for this phenomenon is the increase in the sensitivity obtained using the "gold standard", which allows for the identification of cases with a low infection intensity in these age groups, which could not be detected using a single slide. There was an increase in the positivity rate in over-70-year-old individuals according to the "gold standard" and using one Kato-Katz slide. This increase is probably artificial due to the low number of individuals in these age groups.

According to Gomes et al. (2004), the TF-Test ${ }^{\mathbb{B}}$ technique presents comparable results to the Kato-Katz method in cases of high worm burden. These authors further described that in cases of low worm burden, the positivity was higher using the TF-Test ${ }^{\mathbb{B}}$ than the KatoKatz method. The loss of sensitivity of the Kato-Katz method is due to the technique itself, which utilises only a small faecal sample (41.7 mg of faeces), whereas approximately $4.0 \mathrm{~g}$ of faeces is used in the TF-Test ${ }^{\mathbb{Q}}$. In the present study, different results were found between both techniques. The sensitivity of the TF-Test ${ }^{\mathbb{R}}$ presented comparable values to those of 12 Kato-Katz slides (Table II), but this number of slides corresponded to $500 \mathrm{mg}$ of faecal material compared to the $1,500 \mathrm{mg}$ of faecal material used in the TF-Test ${ }^{\circledR}$. To evaluate the sensitivity of both techniques using the same amount of faecal material, a stool sample from 54 participants was analysed using both techniques using $500 \mathrm{mg}$ of faecal material. The results showed a lower sensitivity of the TF-Test ${ }^{\circledR}$ compared to the Kato-Katz method.

Despite using approximately $1,500 \mathrm{mg}$ of faeces for the TF-Test ${ }^{\circledR}$ and $750 \mathrm{mg}$ for 18 Kato-Katz slides $(41.7 \mathrm{mg}$ per slide), the Kato-Katz technique presented a higher sensitivity. In the Kato-Katz method, the faeces are clarified without any dilution, whereas in the TF-Test ${ }^{\circledR}$, although the quantity of material is two times greater than the Kato-Katz method, some parasite forms are likely lost at the following stages: dilution with $10 \%$ formalin and the use of ethyl acetate to emulsify the faecal material because the supernatant layer $( \pm 0.5 \mathrm{~cm}$ thick, composed of faecal residuals mixed with fat debris) is always discarded (Mendes et al. 2005). The faecal re- 
siduals in the analysed slide in the TF-Test ${ }^{\circledR}$ are large and compound the visualisation of helminth eggs, thus, in our opinion, contributing to its loss in sensitivity. It is also worth noting that on average, 10 slides were necessary, corresponding to $1 \mathrm{~h}$ of work for reading all of the sediments per individual to quantitatively perform the technique. Therefore, the TF-Test ${ }^{\circledR}$ method is not practical when used as a quantitative method due to the time required for microscopy.

The Kato-Katz method also presents some limitations: it is impossible to perform the method using diarrheic samples, it does not detect protozoans, there is a low reproducibility among slides and among different slides of the same individual and dissimilar readings can be obtained by different examiners (Kongs et al. 2001). Another limitation is the analysis of multiple slides because the technicians of the control services usually examine just one slide per individual. For epidemiological purposes, repeated examinations are impracticable and are not economically viable. Thus, in areas with prevalence rates lower than $10 \%$ or in individuals with low worm burdens, it is necessary to use methodologies in combination to obtain levels close to the true prevalence of schistosomiasis.

It is important to emphasise that the Kato-Katz method detected significantly more cases of infection with geohelminths than the TF-Test ${ }^{\circledR}(26.3 \%$ vs. $3 \%)$ and all infections diagnosed using the TF-Test ${ }^{\mathbb{Q}}$ were caused by hookworms.

In conclusion, the TF-Test ${ }^{\circledR}$ presented relatively good results for the diagnosis of schistosomiasis, but the KatoKatz method showed more diagnostic efficacy with an increase in the number of slides and samples collected on different days. The use of both parasitological methods in combination indicates that the prevalence rate in areas of low-transmission is significantly underestimated, in our case by 4.5 times, which has a negative impact on control strategies based on a single slide of a single sample.

Therefore, a more complex diagnostic approach is required under these circumstances, which are characterised by low individual egg counts. One of the alternatives to overcome this lack of sensitivity is, as shown here, an increase in the number of stool samples collected on different days. Another alternative is the combination of different diagnostic methods, including immunological techniques, such as antigen and antibody detection and molecular methods, mainly polymerase chain reaction of faecal, blood or urine samples, which also increases the overall sensitivity to obtain more reliable values for the prevalence of the disease. Because the profile of schistosomiasis is changing, characterised by an increase in low-transmission areas where the current diagnostic tools cannot provide sufficient sensitivity, control strategies need to be adapted adequately to maintain efficacy.

It is important to note that apart from the costs and logistic difficulties caused by the combination of diagnostic techniques for the detection of schistosomiasis cases, the treatment of nearly all infected individuals may result in the elimination of a sufficient number of "providers of miracidiae" to interrupt disease transmission. It is also noteworthy that in Brazil schistosomiasis control is based on repeated parasitological surveys followed by treatments. Currently, in many endemic areas, the diagnosis and treatment of this disease is not a result of a proactive search by public health authorities, but the spontaneous consultation of patients, which can hamper the effective control of transmission in the short term.

\section{ACKNOWLEDGEMENTS}

To Vera de Paula Ribeiro, from the Laboratory of Schistosomiasis of CPqRR/Fiocruz, for the English review, to the cooperation with the technical team of Control Center of Zoonosis, Montes Claros, MG, Brazil, and to the population of Pedra Preta, for their collaboration during the field work.

\section{REFERENCES}

Barreto ML, Smith DH, Sleigh AC 1990. Implications of faecal egg count variation when using the Kato-Katz method to assess Schistosoma mansoni infections. Trans R Soc Trop Med Hyg 84: 554-555.

Bergquist R, Johansen MV, Utzinger J 2009. Diagnostic dilemmas in helminthology: what tools to use and when? Trends in Parasitology 25: 151-156.

Berhe N, Medhin G, Erko B, Smith T, Gedamu S, Bereded D, Moore R, Habte E, Redda A, Gebre-Michael T, Gundersen SG 2004. Variations in helminth faecal egg counts in Kato-Katz thick smears and their implications in assessing infection status with Schistosoma mansoni. Acta Tropica 92: 205-212.

Blagg W, Schoegel EL, Mansour NS, Khalat GI 1955. A new concentration technic for the demonstration of protozoa and helminth eggs in feces. Am J Trop Med Hyg 4: 23-28.

De Vlas SJ, Gryseels B 1992. Underestimation of Schistosoma mansoni prevalences. Parasitology Today 8: 274-277.

Engels D, Sinzinkayo E, Gryseels B 1996. Day-to-day egg count fluctuation in Schistosoma mansoni infection and its operational implications. Am J Trop Med Hyg 54: 319-324.

Enk MJ, Lustosa Lima AC, Drummond SC, Schall VT, Coelho PMZ 2008. The impact of the number of stool samples on the prevalence, the infection intensity and the distribution of the infection with Schistosoma mansoni among a population in an area of low transmission. Acta Tropica 108: 222-228.

Ferrari MLA, Coelho PMZ, Antunes CMF, Tavares CAP, da Cunha AS 2003. Efficacy of oxamniquine and praziquantel in the treatment of Schistosoma mansoni infection: a controlled trial. Bull World Health Organ 81: 190-196.

Gomes JF, Hoshino-Shimizu S, Dias LCS, Araújo AJUS, Castilho VLP, Neves FAMA 2004. Evaluation of a novel kit (TF-Test) for the diagnosis of intestinal parasitic infections. J Clin Lab Anal 18: 132-138.

Katz N, Chaves A, Pellegrino J 1972. A simple device for quantitative stool thick-smear technique in schistosomiasis mansoni. Rev Inst Med Trop Sao Paulo 14: 397-400.

Kongs A, Marks G, Verlé P, Van der Stuyft P 2001. Limitations of Kato-Katz technique for evaluating $S$. mansoni infections. Trop Med Int Health 6: 163-169.

Landis JR, Koch GG 1977. The measurement of observer agreement for categorical data. Biometrics 33: 159-174.

Lin DD, Liu JX, Liu YM, Hu F, Zhang YY, Xu JM, Li JY, Ji MJ, Bergquist R, Wu GL, Wu HW 2008. Routine Kato-Katz technique underestimates the prevalence of Schistosoma japonicum: a case study in an endemic area of the People's Republic of China. Parasitoly Int 57: 281-286.

Mendes CR, Teixeira AT, Pereira RA, Dias LC 2005. A comparative study of the parasitological techniques: Kato-Katz and coprotest. Rev Soc Bras Med Trop 38: 178-180. 
Teles HMS, Ferreira CS, Carvalho ME, Zacharias F, Magalhães LA 2003. Eficiência do diagnóstico coproscópico de Schistosoma mansoni em fezes prensadas. Rev Soc Bras Med Trop 36: 503-507.

Utzinger J, Booth M, N'Goran EK, Muller I, Tanner M, Lengeler C 2001. Relative contribution of day-to-day and intra-specimen variation in faecal egg counts of Schistosoma mansoni before and after treatment with praziquantel. Parasitology 122: 537-544.
WHO - World Health Organization 1993. The control of schistosomiasis, Technical Report Series 830, WHO, Geneva, p. 1-85.

Zhang YY, Luo JP, Liu YM, Wang QZ, Chen JH, Xu MX, Xu JM, Wu J, Tu XM, Wu GL, Zhang ZS, Wu HW 2009. Evaluation of KatoKatz examination method in three areas with low-level endemicity of schistosomiasis japonica in China: a Bayesian modeling approach. Acta Tropica 112: 16-22. 